\title{
Clinicopathological Case Conference: A 17 year old with progressive gait difficulty and a complex family history
}

\author{
David Anderson', Bonny Cheyip', Jeannine Heckmann ${ }^{2}$, Myles Connor ${ }^{1}$ \\ ${ }_{2}^{2}$ Division of Neurology, Department of Neurosciences, University of the Witwatersrand, Johannesburg, South Africa \\ ${ }^{2}$ Division of Neurology, Groote Schuur Hospital and University of Cape Town, Cape Town, South Africa
}

\section{This case was presented and discussed at the Annual Neurology Course, held in Johannesburg, October 2004. The content represents a narrative account of the actual presentation and discussion.}

Keywords: Clinical conference, Genetics, Spastic paraparesis

Received: 27.01 .05

Accepted: 07.03 .05

\section{Case presentation}

A 17 year old (BM) presented for the first time in 2004 accompanied by her maternal grandmother. Over the past 3 years she had experienced insidiously progressive "fatigue" in her legs on walking. She complained of tiring easily and said that children at school laughed at her because of her "funny" gait. She had never been able to play sport. She stopped attending school 2 years ago because she was no longer able to walk the long distance to school, difficulty in climbing stairs at school and the embarrassment of being ridiculed at school. She could now only manage to walk short distances. She had passed grade IX and had not experienced any learning difficulties.

BM was apparently born 2 months prematurely and spent the first 2 months in hospital. Thereafter she developed normally, and was able to walk and talk at appropriate times according to her grandmother. At 5 months of age she was accidentally dropped and the family attribute her current problem to this incident.

She also complained of deep pain in the right leg but no paraesthesiae or back pain. There were no bladder or bowel symptoms or symptoms related to her arms. On direct questioning she admitted to depressed mood, insomnia and tearfulness.

BM did not have any other contributory past medical and surgical history. She did not have allergies and did not smoke or drink alcohol. She said that she had a boyfriend and wanted to start contraception.

On direct questioning she revealed a family history of neurological disease. Her younger half-sister TM, aged 12 (different father), had been paralysed from the hips down since "early childhood" and was confined to a wheelchair. TM attended a school for cerebral palsied children and was followed at a paediatric neurological clinic for her problems associated with "cerebral palsy". She had another half-sister KM, aged 1, from a 3rd father, who was born 2 months

\section{Correspondence:}

Dr MD Connor, Division of Neurology, Department of Neurosciences,

University of the Witwatersrand.

email: connormd@medicine.wits.ac.za prematurely; KM had an abnormal foot and had delayed developmental milestones.

Her mother (aged 37) had seizures as a small child, but has not suffered from seizures off medication for many years. However, the grandmother remarked that her daughter was emotionally labile and recently (over the past 2 months), people had commented that she sometimes walked as if she were drunk. The family was unaware of a drinking problem. Her maternal grandmother, aged 56, had hypertension and apparently suffered two strokes (1990 and 1996) and her maternal grandfather died in his 30's with hypertension.

Her general examination was normal with a regular pulse of 80 per minute and a blood pressure of 110/70 $\mathrm{mmHg}$. She had no evidence of lymphadenopathy or thyroidmegaly. The respiratory, cardiovascular and abdominal system examinations were unremarkable.

Higher functions were not formally tested but the patient gave a good account of herself with a careful, clear history. The pupils were equal and reactive to light. The optic discs appeared normal on fundoscopy. The extra-ocular eye movements were normal and the cranial nerves were intact. Upper limb examination showed normal power, tone, reflexes, sensation and co-ordination.

There was mild wasting of the distal lower limbs. The tone was increased at the knees. There was evidence of a mild upper motor neurone pattern of weakness; hip flexors 4 - to 4 , knee flexion 4 with the remainder at $5 / 5$. The reflexes were brisk at the knees but the ankles were barely present. The plantar responses were extensor. The sensory modalities of touch and pain were normal in the legs. There was minimal proprioceptive loss with fine toe movement $(8 / 10$ responses correct). Co-ordination was normal in the upper and lower limbs. The gait was broad-based with stiff legs and evidence of bilateral circumduction.

There was no evidence of musculoskeletal abnormality and no spinal tenderness.

Laboratory investigations revealed a haemoglobin of 12.2 (13-17 $\mathrm{g} / \mathrm{dl})$, white cell count of $9.9(4-10 \times 109 / \mathrm{L})$ and platelet count of 420 (137-373 x109/L); the differential blood count and smear were normal. The erythrocyte sedimentation rate 19 (0-31 in $1 \mathrm{hr})$. The thyroid stimulating hormone was not performed (possibly the result of 
insufficient blood for testing) and the T4 was 10.5 . The serum sodium was $136 \mathrm{mmol} / \mathrm{L}(135-147 \mathrm{mmol} / \mathrm{L})$, potassium $4.6 \mathrm{mmol} / \mathrm{L}$ (3.3-5.3 mmol/L), creatinine $60 \mu \mathrm{mol} / \mathrm{L}(60-120 \mu \mathrm{mol} / \mathrm{L})$ and urea $2.1 \mathrm{mmol} / \mathrm{L}(2.6-7.0 \mathrm{mmol} / \mathrm{L})$. The serum HTLV-1\&2 antibody screen was negative. A serum vitamin B12 was 293 pmol/L (133-675 pmol/ L).

The ECG was normal. A lumbar spine and chest X-ray were normal. The T1- and T2-weighted images on MRI of the cervical and thoracic cord were normal. The FLAIR image of the brain showed mild, patchy, ill-defined signal changes in the peri-ventricular white matter of the occipital horns of the lateral ventricles and centrum semi-ovale on the right.

The paediatric neurologist looking after TM mentioned that she had heard BM was seen at the adult clinic. Despite TM's diagnosis of "cerebral palsy" for more than 8 years they were concerned as she recently started complaining of visual difficulties. On re-examination of TM recently she appeared to be losing her lower limb reflexes.

A diagnostic procedure was performed......

\section{Discussion}

\section{DrD Anderson and Dr B Cheyip}

\section{Clinical features}

We will start by summarising the key features of BM's clinical presentation. She presented complaining of an insidiously progressive 'fatigue' in her legs on walking which was most noticeable over the last three years. This was severe enough to prevent her walking to school now and had also been noticed by other children at school resulting in her being ridiculed for having a funny walk. She had no associated back pain, no bladder and bowel problems and no paraesthesiae. She complained of insomnia and depressed mood.

Her past medical history included being born prematurely with a two month hospital stay. However, she then apparently achieved her milestones normally and has passed grade IX and has no obvious learning difficulties. Although she was dropped at 5 months of age it is not clear that this has any direct bearing on her current problems.

The most striking feature of BM's history is her family history.

Although we are not told exactly how many siblings she has, we are told that she has two half sisters from different fathers and both of them seem to have either clear cut or potential neurological problems.

TM, aged 12, is thought to have cerebral palsy and was paralysed since early childhood. However she has recently been noted to have developed visual difficulties and has begun to lose reflexes. KM aged 1, was born 2 months premature with an abnormal foot (possibly a clubbed foot) and has delayed milestones.

BM's mother is 37 years old and had seizures as a child. She is considered emotionally labile and recently her gait has become ataxic with no clear history of alcohol use. BM's grandparents both had hypertension and her maternal grandmother had suffered two strokes.

BM's general examination including musculoskeletal examination was normal. Her higher functions although not fully assessed appeared normal. Her cranial nerves were normal including the optic nerves. Unfortunately we are not told about the rest of the fundoscopic examination and the appearance of the retina. The upper limb examination was normal, but the lower limbs revealed an upper motor pattern weakness with spasticity and she had an extensor plantar response. Although the sensory examination was normal for light touch and pain, there was possibly minimal proprioception impairment and she had decreased ankle reflexes. Her gait was broad based and spastic. Romberg's sign is not mentioned.

Her blood investigations were normal as were the ECG and radiology as mentioned. The MRI of her spine was normal, but on FLAIR images of her brain a few mild patchy periventricular white matter changes were visible asymmetrically.

In assessment at this point we think that this is probably an inherited disorder affecting primarily the females of this family. There is a wide variation of clinical presentation though, including: spastic paraparesis, mental retardation and cognitive impairment, neuropathy, visual involvement and MRI white matter changes.

To further clarify what disorder this might be we will consider the inheritance pattern.

\section{Genetic patterns}

Although genetic patterns include autosomal dominant, autosomal recessive, X-linked, mitochondrial, repeat expansion disease and polygenic patterns, we will briefly highlight the patterns that could most likely explain a complex family pedigree such as this one (Figure 1).

Autosomal dominant disorders are caused by a single disease copy of the gene i.e. a mutation in one allele (of one chromosome) of the diseased gene. Heterozygotes manifest the disease and both males and females may be affected. Penetrance may be variable. Phenotypes may also differ in individuals with the same genetic disorder due to genetic imprinting in which differences in expression of maternal and paternal alleles result in differing phenotpyes.

Diseases due to DNA repeat expansions may present with dominant, recessive or X-linked inheritance patterns and primarily present as neurological disorders. Mutations are dynamic and change in size from generation to generation most often getting bigger in subsequent generations. Clinically this is seen as the disease often being more severe and with earlier onset in subsequent generations such as seen myotonic dystrophy.

Mitochondrial disease results from mutations in genes within the mitochondrial genome. Mutations are maternally inherited and the manifestation of disease depends on the ratio of mutant to wild-type mitochondria (heteroplasmy). Both males and females are affected but males cannot transmit the mutations to offspring. Examples include MELAS (mitochondrial encephalopathy, lactic acidosis, and stroke), Kearns-Sayre syndrome, NARP (Neuropathy, Ataxia, Retinitis Pigmentosa Syndrome), MERRF (myoclonic epilepsy and ragged red fibers in muscle)..

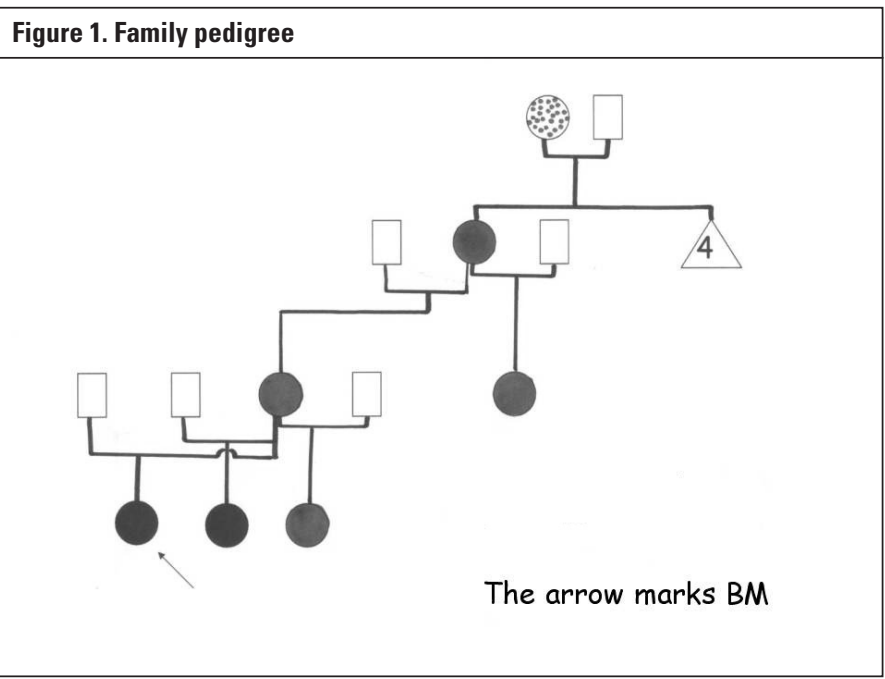




\section{Differential diagnosis}

Using the most significant clinical feature of our index case BM, that of a hereditary spastic paraparesis, we consider the following differential diagnosis:

Hereditary spastic paraparesis, leukodystrophy (including adrenomyeloneuropathy), Krabbe's disease, metachromatic leukodystrophy, Dopa-responsive dystonia, and mitochondrial disease. Hereditary spastic paraparesis is a syndromic designation for a group of inherited disorders in which the main symptoms are progressive lower limb spasticity and weakness. There is variable age at onset. Associated features include: urinary urgency, pes cavus and mildly impaired vibration sense and 'complicated' forms may include dementia, seizures, extrapyramidal signs, cutaneous abnormalities, cataracts, peripheral neuropathy and amyotrophy. There are many types (SPG 1 through 17) and inheritance is heterogeneous and may be autosomal dominant, recessive, X-linked or even mitochondrial. MRI white matter changes have been described.

The leukodystrophies are defined as inherited abnormalities of myelin metabolism affecting both the central and peripheral nervous systems. Adrenoleukodystrophy is an X-linked condition which presents with adrenal insufficiency, progressive myelopathy, peripheral neuropathy and encephalopathy. It affects 1:20 000 male births and is caused by peroxisomal dysfunction resulting in abnormal betaoxidation of very long chain fatty acids. However, heterozygous females with chronic spastic paraparesis and little if any adrenal insufficiency have been described. ${ }^{1} \mathrm{Up}$ to half of these heterozygote females have white matter changes on MRI.

Metachromatic leukodystrophy may present with spastic paraparesis at least initially, loss of vision and cognitive decline. It is caused by aryl sulfatase deficiency. It is inherited in an autosomal recessive pattern which makes it unlikely in our family.

Krabbe disease caused by galactocerebrosidase deficiency presents with generalised rigidity, irritability, neuropathy, blindness and convulsions but usually occurs in children and results in death by about two years of age.

Mitochondrial disorders show much heterogeneity in both clinical features and mode of inheritance. The diseases are often show multisystem involvement in which neurological dysfunction predominates (central and peripheral), myopathies, deafness and endocrinopathy (diabetes and short stature). Of specific interest in our family though are the Leigh Disease or subacute necrotising encephalomyelopathy and NARP (Neuropathy Ataxia Retinitis Pigmentosa) syndromes. These two conditions share the same mitochondrial gene mutation. In Leigh disease clinical features include poor head control, hypotonia, and myoclonic jerks in infants. If the onset is in the second year of life then tonic spasms, difficulty walking, external ophthalmoplegia and ataxia are seen. In milder and more protracted cases, the clinical picture is often mistaken for cerebral palsy and peripheral neuropathy may develop in some cases. Brain imaging may show lesions of the lenticular nuclei and brainstem but MRI white matter changes have been described.

NARP presents with developmental delay, neuropathy, ataxia and retinitis pigmentosa with visual loss and only rarely develops after the age of 20. There is great heterogeneity though and indeed even within a particular family the most severely affected individual may present with a severe phenotype of Leigh Disease, and the least affected with a mild form of NARP.

\section{Summary}

We think that the patient and her family had either NARP / Leigh type mitochondrial disease, or less likely were heterozygotes with adrenoleukodystrophy. We think that the investigation was a muscle biopsy to look for evidence of mitochondrial disease .

\section{Presentation of investigation findings and comment Dr J Heckmann}

We took EDTA blood for mitchondrial genomic analysis. Essentially, after DNA extraction certain common sites where mutations are known to cause disease, are amplified using PCR. The index patient and two other family members had the mitochondrial DNA 8993 (NARP) mutation.

\section{Discussion}

As the discussants quite correctly pointed out this particular mutation may present as a more severe Leigh's encephalopathy or "milder" NARP variants. ${ }^{2}$ However, anticipation occurs, as in this family, where the disease appears to become more severe and with earlier age at onset in successive generations. ${ }^{2}$ NARP may initially be mistaken for cerebral palsy and should always be considered as a possibility in unexplained developmental delay or spastic paraparesis. ${ }^{3,4}$ Some families may show significant variability as in this family; those with the Leigh's phenotype have a very high proportion ( $>95 \%)$ of mutant mitochondrial DNA (mtDNA) and those with the NARP phenotype much lower levels $(>60 \%)$ of mutant mtDNA lymphocytes. ${ }^{2}$ Some family members with low mutant mtDNA levels may even be norma. ${ }^{2}$

Muscle biopsies do not always show the typical features of mitochondrial dysfunction and this is particularly relevant in NARP. $^{3}$ The diagnostic method of choice is to confirm a clinical suspicion of a mitochondnial disorder with mtDNA analysis. Unfortunately in South Africa only a few common mutations are screened for currently.

\section{Conclusion}

In conclusion, this family had the NARP T8993G mtDNA mutation. ${ }^{4}$ As often occurs, there was marked clinical heterogeneity of the phenotype within the family; this ranged from developmental delay, myeloneuropathy, progressive degenerative spinocerebellar degenration and dementia, to late-onset retinits pigmentosa with mild spastic paraparesis. The family benefited from counselling, paramedical intervention and certain members have responded to antidepressant medication.

\section{Acknowledgements:}

We would like to thank Dr EP Owen from Chemical Pathology, University of Cape Town for the molecular analysis and Dr G Riordan from Red Cross Children's Hospital who referred the non-pediatric members of this family for evaluation.

\section{References}

1. Moser HW. Adrenoleukodystrophy: phenotype, genetics, pathogenesis and therapy. Brain 1997;120(Pt 8):1485-508.

2. Uziel G, Moroni I, Lamantea E, Fratta GM, Ciceri E, Carrara Fet al. Mitochondrial disease associated with the T8993G mutation of the mitochondrial ATPase 6 gene: a clinical, biochemical, and molecular study in six families. J Neurol.Neurosurg.Psychiatry 1997;63(1):16-22.

3. Fryer A, Appleton R, Sweeney MG, Rosenbloom L, Harding AE. Mitochondrial DNA 8993 (NARP) mutation presenting with a heterogeneous phenotype including 'cerebral palsy'. Arch.Dis.Child 1994;71(5):419-22.

4. Santorelli FM, Mak SC, Vazquez-Memije E, Shanske S, Kranz-Eble P, Jain KD et al. Clinical heterogeneity associated with the mitochondrial DNA T8993C point mutation. Pediatr.Res 1996;39(5):914-7. 